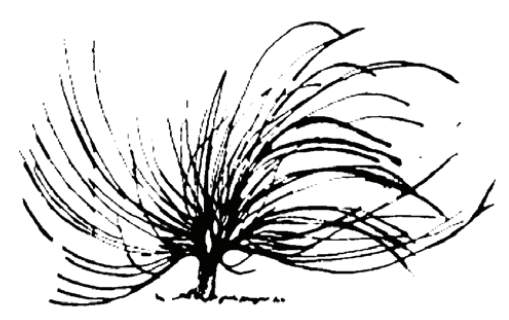

\title{
Una Propuesta Didáctica para el Desarrollo de la Destreza Oral en Inglés en Estudiantes de Décimo y Undécimo Año de Colegios Públicos
}

\author{
Yamileth Moreira González ${ }^{1}$ \\ Universidad de Costa Rica \\ San José, Costa Rica \\ yamileth.moreira@gmail.com
}

\section{Resumen}

El objetivo principal de este artículo es proveer una propuesta didáctica para los docentes que imparten inglés como lengua extranjera en colegios académicos, públicos de secundaria, con el fin de fortalecer la producción oral de la lengua inglesa de sus estudiantes y que estos participen en conversaciones de la vida diaria o sobre diferentes temas de interés relacionados con la sociedad costarricense. Un objetivo es fomentar el uso de estrategias orales participativas y transaccionales al abordar situaciones que requieran la producción oral precisa que garantice un entendimiento mutuo. El otro es incrementar el vocabulario y la lengua técnica, formal e informal para situaciones, tanto laborales como cotidianas. Las estructuras lingüísticas se presentan para cada tema del programa del Ministerio de Educación Pública en los niveles de décimo y undécimo como ejemplo para los

\section{(c) (i) $(-)$}

Recibido: 3 de mayo de 2015 - Aprobado: 18 de agosto de 2015

1 Profesora de inglés en la Escuela de Lenguas Modernas, UCR. Magíster en la Enseñanza del Inglés como Lengua Extranjera. Licenciada en Lingüística, UCR. Bachiller en Inglés UCR. 
docentes. A la vez, se dan muestras de situaciones que los guiarán para desarrollar otras de acuerdo con las necesidades del grupo y también con la creatividad de los mismos estudiantes.

Palabras claves: hablar, inglés, estudiantes de secundaria, educación pública, secundaria, situaciones orales, vida cotidiana costarricense

\begin{abstract}
The main objective of this article is to provide teachers who teach English as a foreign language in public academic schools with a teaching approach to strengthen the oral production of English in tenth and eleventh graders by using the content of the syllabus of the Education Ministry (MEP). One specific objective of this is to encourage the use of participatory and transactional oral strategies to address daily life situations to interact and get a mutual understanding. The other one is to increase vocabulary and technical, formal and informal language to be used in workplaces and everyday situations. Linguistic structures are presented in every level and content included in the tenth and eleventh levels as well as sample situations that will lead English teacher to develop other situations based on the needs of the group and the creativity of the students.
\end{abstract}

Key words: speaking English, high school students, public education, oral situations, Costa Rican daily life

\title{
ANTECEDENTES
}

Atendiendo a la Política Educativa hacia el Siglo XXI, aprobada en 1994 por el entonces Ministro de Educación Eduardo Doryan (2010), en que se hace un esfuerzo innovador para establecer un marco de largo plazo para el desarrollo del Sistema Educativo Costarricense, que permite adecuar el mandato constitucional a la realidad específica de nuestros días, he decidido unirme a este esfuerzo para formar recursos humanos que eleven la competitividad del país, necesaria para 
triunfar tanto en los mercados nacionales como internacionales. Junto a esta visión humanista, racionalista y constructivista urge al sistema integrarse curricularmente para lograr una globalización, esto es:

Integrar el currículo y en específico utilizar el enfoque interdisciplinario que permite al profesor explicar cómo intervienen y se relacionan tres elementos principales: profesor, estudiante y contenido para crear las condiciones idóneas que favorecen la construcción de un aprendizaje significativo. (Quintana, 1998, pp. 1,2)

Quintana (1989) enfatiza el hecho de que no es sólo enseñar temas a los estudiantes, es integrar esos temas en varios enfoques, en los cuales Gaff (citado por Quintana, 1989) establece tres argumentos que incluyen además de la adquisición del conocimiento de otros campos, la integración de ese conocimiento en forma integral tomando en cuenta el aspecto social. De estos, el primero es el intelectual indicando que "los que promueven la integración del currículo aducen que las ideas en cualquier campo de estudio se enriquecen con las teorías, conceptos y conocimientos de otros campos (p.1)." El segundo es el pedagógico donde el conocimiento se presenta integrado y no aislado: "Un aprendizaje auténtico se logra sólo si el mismo posee unos atributos tales como: relación con el mundo, unidades temáticas y el desarrollo de las competencias en vez de destrezas aisladas (p.1)." $\mathrm{Y}$ el tercero es el argumento social en el cual "el aprendizaje es una actividad individual, pero esto no significa que sea solitaria" (p.1). Este es más efectivo cuando el profesor convierte su salón de clases en una comunidad de aprendizaje, en la cual todos aportan al desarrollo intelectual de los compañeros. Los cursos diseñados de forma integrada por lo general interesan más a los estudiantes y les ofrecen temas más interesantes para conversar fuera del salón de clases precisamente porque son más relevantes al mundo en que vivimos.

Los educadores que tienen a su cargo el desarrollo curricular no pueden cerrarse a los cambios sociales, económicos y políticos que están ocurriendo. Por eso, este artículo propone otorgar al estudiante una herramienta más que le proporcionará la facilidad para su futura inserción en la fuerza laboral. Johnston (1991) visualizó en esa década cómo sería la movilidad laboral en la que los trabajadores deberán tener las destrezas necesarias para desempeñarse mejor. Este fenómeno se está viviendo 
actualmente con las compañías transnacionales que urgen empleados que hablen el inglés para lograr desarrollarse en su campo.

El Proyecto Costa Rica Multilingüe (2012) empezó en el 2007, como una necesidad de incrementar en la población costarricense las habilidades comunicativas en la lengua inglesa, para una mejor inserción en el campo laboral y una mejor competitividad. Es así como a partir del 2008, con la Estrategia Siglo XXI, con la alianza de organizaciones como la Casa Presidencial, el Ministerio de Educación, el Ministerio de Comercio y Economía y CINDE (Coalición de Iniciativas de Desarrollo), empiezan las capacitaciones para el INA (Instituto Nacional de Aprendizaje) y profesores del Ministerio de Educación Pública que ayudarían al estudiantado a alcanzar un nivel $\mathrm{C} 1$ dictado bajo el marco de la Comunidad Europea en referencia a las lenguas.

\section{JUSTIFICACIÓN}

Algunos estudiantes de secundaria que se gradúan como bachilleres, quieren buscar trabajo por varias razones, por ejemplo, ayudar económicamente a la familia, solventar sus gastos personales y poder pagarse una educación superior. Por medio de la presente propuesta metodológica, se pretende que los alumnos mejoren la destreza oral en inglés, lo cual les ayudará a desempeñarse de una manera adecuada en los empleos donde se requiera que el trabajador sea bilingüe (inglés-español) como centros de servicio, hoteles, tiendas de artesanías, cafeterías y restaurantes más frecuentados por los turistas. De esta manera, los jóvenes se integran al mundo laboral en procura de una estabilidad económica que será efecto multiplicador en el mejoramiento de la familia y de la comunidad donde viven.

Los estudiantes sienten y tienen una gran necesidad al graduarse de hablar el inglés en forma fluida, de tal forma que puedan obtener un mejor trabajo y a la vez desarrollarse personal y profesionalmente. Este artículo pretende dar a conocer a la población estudiantil y al profesorado, la importancia de usar situaciones de la vida real con el propósito de sostener una conversación en inglés.

Actualmente, los programas de décimo y undécimo año están enfocados en preparar a los estudiantes para exámenes de bachillerato, evaluándoles solamente en la destreza y aplicación de las estrategias de lectura. Debido a esto, la habilidad oral se ha minimizado y 
los estudiantes no desarrollan realmente los objetivos a alcanzar en la lengua inglesa.

Este artículo pretende guiar a los profesores de colegios públicos dándoles ejemplos de situaciones orales que podrán usar en el aula para que al obtener su bachillerato los estudiantes tengan la habilidad de hablar en inglés para desenvolverse en diferentes trabajos que así lo requieran.

Las situaciones que se presentan proveerán las herramientas necesarias a los docentes para que los futuros graduandos de colegios académicos públicos de secundaria a través de un fortalecimiento de la producción oral de la lengua inglesa les permitan entablar conversaciones de la vida diaria o sobre diferentes intereses relacionados con la sociedad costarricense. Estas situaciones fomentan el uso de estrategias orales participativas y transaccionales al abordar situaciones que requieran la producción oral precisa que garantice un entendimiento mutuo. Al mismo tiempo, incrementan el vocabulario y la lengua técnica, formal e informal para situaciones tanto laborales como cotidianas.

\section{MARCO TEÓRICO}

El enfoque oral en que se daban situaciones al estudiante para aprender una segunda lengua empezó a desarrollarse por la lingüística aplicada británica en la década de 1930 hasta la década de 1960. Principalmente, consistía en que las estructuras gramaticales y el vocabulario frecuente que posiblemente se usan en lecturas, se presentaban en situaciones de la vida real en las que podían ser usadas. Este enfoque tiene que ver con acondicionamiento de los estudiantes en procesos de aprendizaje que los llevará a aprender exitosamente. Aunque en la actualidad, el profesor usa varias metodologías para enseñar la segunda lengua, los profesores de inglés como segunda lengua e inglés como lengua extranjera coinciden en que la estructura de la lengua y el discurso se entrelazan para lograr desarrollar la lengua hablada.

Los lingüistas británicos enfatizaron que la segunda lengua debe ser presentada con las estructuras que deben usarse distinguiendo el enfoque oral situacional. A la vez, mencionaron la importancia del vocabulario a través de la lectura para ayudar al estudiante a internalizar las reglas y estructuras de la oración. Es el educador el que provee las funciones y nociones en situaciones reales para darle énfasis a los propósitos comunicativos del acto del discurso (Finocchiario y 
Brumfit, 1983). Los estudiantes deben ser conscientes del conocimiento para luego aplicarlo en situaciones reales (Schmidt, 1990). Muy ligado a estas estructuras gramaticales está el vocabulario que debe usar el hablante de la segunda lengua en esas funciones. Aunque la teoría de la adquisición del vocabulario de una segunda lengua demuestra que éste se adquiere indirectamente a través de la lectura, no hay certeza de que este vocabulario lo utilicen los estudiantes en forma oral y efectiva en ciertas situaciones (Zimmerman, 1997). Esta necesidad nos lleva a plantear esta metodología.

De ahí que, esta metodología le da a los estudiantes la oportunidad de usar el vocabulario y las estructuras lingüísticas dadas por el docente en diferentes situaciones del diario vivir. Algunos lingüistas creen que cuanto más vocabulario aprenda un estudiante de la lengua meta es mejor. Hay vocabulario básico, como palabras de alta frecuencia, así llamadas por como artículos, preposiciones, verbos frecuentes, palabras técnicas y aquellas palabras que se parecen a la lengua nativa que siempre lo van a usar (Nation, 2001). De ahí, que no es la cantidad de vocabulario sino el uso de este vocabulario que les ayudará a desarrollar la segunda lengua. Este vocabulario debe presentarse en forma sistemática (Reyes, 2004-05) para favorecer su uso en la clase.

El educador debe diseñar el material para ese propósito y los estudiantes valoran la competencia interaccional, o las competencias necesarias para eventualmente usarlas en conversaciones basadas en la investigación sobre el tema para hacer las conversaciones más efectivas (Barraja-Rohan, 2011). Igualmente, el estudiante puede participar en la evaluación de la metodología siguiendo los criterios dados por el profesor. Por lo tanto, una rúbrica bien elaborada por ambas partes, darán los mejores resultados, ya que los estudiantes serán capaces de detectar y autocorregirse cuando están hablando. De ahí que la evaluación se hace más asertiva y los estudiantes reaccionan en forma positiva al proveérseles con opciones apropiadas para que muestren su conocimiento, habilidades y comprensión (Mc.Thiegh y O'Connor, 2009, p.177).

La integración de destrezas en el aprendizaje de una segunda lengua es esencial. Las habilidades de audición, comprensión de lectura, habla y escritura se integran para que el aprendizaje sea eficaz. Para este proceso de aprendizaje, el docente, provee a los estudiantes con videos y lecturas como actividades previas al desarrollado de las actividades orales. 


\section{METODOLOGÍA}

Entre los objetivos de esta propuesta metodológica con situaciones orales están la integración de las cuatro habilidades de una lengua a través de la estructura de la lengua, la exactitud de la pronunciación y la habilidad de responder de forma rápida y exacta a situaciones orales. Este enfoque tiene la ventaja de que en corto tiempo el docente puede ver los resultados de los estudiantes usando las estructuras en determinadas situaciones. A través de la repetición y formación de estructuras de la lengua, ellos desarrollan habilidades para adquirir la competencia de esas estructuras. Dicha metodología puede dar excelentes resultados, especialmente en los colegios públicos donde hay carencia de laboratorios y grabadoras y en que la única fuente de pronunciación y proveedor de lecturas o material audiovisual es el docente mismo que los guía para adquirir la competencia en la lengua.

El vocabulario consiste en palabras o frases de uso frecuente en el tema y las estructuras lingüísticas no son tan complejas ya que están hechas para comunicarse en determinadas situaciones. Este vocabulario se basa en los contenidos de los programas dados por el Ministerio de Educación Pública. La tarea del docente es introducir temas con lecturas de periódicos o revistas para que los estudiantes al comunicarse en forma oral usen aquellas palabras básicas para el tema en discusión y adquieran confianza en su uso.

Las actividades se deben realizar en pares o en grupos pequeños dentro del aula, lo que proporcionará una realimentación de compañero a compañero bajo la supervisión del profesor. El profesor podrá usar las situaciones como evaluaciones orales que le dará un resultado final sorprendente para ambos: profesor y alumno

Con esta metodología se puede tener la oportunidad de contribuir a los esfuerzos del Ministerio de Educación en el ámbito nacional, para que los futuros graduandos puedan realizar conversaciones y tener todo el material informativo (input) para desarrollarse en el campo laboral.

El papel del docente no es solo guiar, más bien, es despertar en los estudiantes la adquisición del conocimiento de una forma activa. Siendo el docente un facilitador, los alumnos pueden desarrollar su propio proceso de aprendizaje. En todo momento, el docente los estará realimentando en algunos aspectos como la pronunciación y uso correcto de estructuras del lenguaje. 
Se ha dividido este proceso en tres etapas: la primera es proporcionar el vocabulario necesario a los estudiantes para desarrollar las situaciones orales que se les dará, la segunda etapa es introducir las estructuras básicas que ellos deben usar en cada situación oral y la última etapa son las situaciones en las que tendrán que usar ese lenguaje y vocabulario.

\section{Primera etapa}

Utilizando la lengua meta, el docente presenta el vocabulario básico y los estudiantes lo trabajan usando el diccionario manual o digital, para que así conozcan su significado. Para una mejor comprensión del vocabulario, el docente lo pronuncia sirviendo de modelo para que estos lo repitan y practiquen correctamente. Además, puede hacer uso de posters manuales o digitales para mostrarles el vocabulario en oraciones; también, darles una lectura corta que incluya el vocabulario y la lengua básica, una grabación corta, o bien, un video para motivarlos y que reconozcan el vocabulario en contexto. Se mencionan algunos sitios donde encontrarán videos o lecturas cortas para la motivación. Los contenidos de décimo y undécimo años son los enviados a las instituciones públicas de secundaria en el nivel nacional (Ver Apéndice A).

\section{Segunda etapa}

El docente presenta las estructuras de la lengua que deben usar en las situaciones. Cada estudiante participa con su compañero de par usando las estructuras en diferentes oraciones, o bien, el docente puede usar algunas estructuras repitiéndolas en oraciones simples y que ellos produzcan otras más complejas.

\section{Tercera etapa}

Esta etapa se considera como la más importante y productiva. Los estudiantes toman una situación oral preparada con antelación, revisan los ejemplos y empiezan a desarrollarla usando correctamente el vocabulario y las estructuras que ya han sido practicadas en clase. 
Las situaciones deben ser de la vida real, auténticas y creativas para que despierten en los estudiantes mucha motivación. El profesor debe ser cuidadoso al escogerlas. Es aconsejable que el docente coloque en cartulina y plástico las situaciones, ya que estas son un reto para los estudiantes, por tanto, la manipulación las puede dañar por factores externos como el nerviosismo y la ansiedad al hablar y causar daños físicos en el papel como el arrugamiento por el doblez involuntario. La idea es que esas situaciones se vuelvan a usar, ya sea como repaso con el mismo grupo, o bien, en otros grupos. Se proveen actividades previas al uso de las situaciones orales, se les da una lista de vocabulario como ejemplo para décimo año (Apéndice B) y undécimo año respectivamente (Apéndice C).

En este punto, el docente observa y ayuda en algunas palabras o estructuras que los estudiantes todavía no conocen. Si están trabajando en pares o en grupos, el docente les pide a los estudiantes que roten para que puedan desarrollar las diferentes situaciones con otros compañeros.

Para la escogencia de las estructuras gramaticales que van a usar los estudiantes, el profesor debe planearlas y ponerlas impresas en tarjetas o fotocopias para cada par de estudiantes, o bien, puede presentarlas en PowerPoint (los que tengan acceso) para practicarlas antes de empezar a dar las situaciones.

\section{Tiempo lectivo}

Tomando en cuenta que las horas lectivas de los estudiantes en secundaria son de 40 minutos y que reciben cinco lecciones semanales en colegios públicos, se considera que la primera etapa puede desarrollarse en esos cuarenta minutos, mientras que para las dos etapas siguientes se necesitan los 80 minutos de las clases restantes de la semana. Este es un tiempo probable y depende de la manera cómo el docente presente el vocabulario. Para desarrollar la estrategia, en total se necesitan tres lecciones dependiendo de las actividades previas como lecturas o videos que el profesor les haya dado. En las clases sucesivas, ya los estudiantes tienen un bagaje cultural y lenguaje básico que lo usará en todas las situaciones futuras, o bien, reciclará ese conocimiento llevándolo a reforzar conocimiento e ir más allá de lo requerido. 


\section{Posibles debilidades de la propuesta}

Entre las posibles debilidades del método están el tiempo y la motivación. El proceso debe hacerse en el tiempo previsto, de ahí que el docente debe estar muy consciente de la gran responsabilidad que tiene durante todo el proceso. No debe apresurar a los estudiantes pero sí llevarlos de la mano para que produzcan lo que se pretende. Los estudiantes quieren desarrollar las situaciones en forma rápida y se desmotivan. El docente debe advertirles que todo depende de cuánto empeño ellos pongan en hacer las primeras etapas para ver buenos resultados. Los profesores deben estar conscientes de que dependiendo del grado de motivación que ellos proporcionen a los estudiantes, así serán de fructíferos los resultados.

\section{Los beneficios variados y satisfactorios de la propuesta}

Los estudiantes enriquecen su vocabulario en la segunda lengua. Como las situaciones orales están basadas en el lenguaje en inglés que se desarrolla en diferentes situaciones de la vida real, ellos están muy motivados y a veces van más allá de lo que el docente pueda imaginarse.

Por otro lado, los estudiantes adquieren un gran bagaje cultural, pues ellos estarán en contacto con diferentes situaciones culturales como tradición, comida, lengua coloquial, turismo en nuestro país y otros que les darán la oportunidad de comparar y reconocer la importancia de las culturas alrededor del mundo, así también la valoración de su propia cultura al compararla con otras.

Además, los estudiantes adquieren fluidez en la lengua meta. Ellos tendrán las herramientas necesarias para escuchar, entender y mantener una conversación en la lengua inglesa llevándolos a ir más allá del tema en cuestión.

También, la confianza y la autoestima aumentarán. Los estudiantes desarrollarán más conocimientos y análisis del uso de la lengua en determinadas situaciones que los hará desarrollar una mejor autoestima al ser capaces de transmitir conocimiento en una segunda lengua.

Del mismo modo, habrá un ambiente excelente de socialización e integración en el aula. Los estudiantes tendrán más oportunidades de hablar, conocer y socializar en el aula manteniendo una conversación en inglés que les dará confianza en sí mismos. Los estudiantes mostrarán 
orgullo de demostrar lo que saben. Se motivarán al hablar la segunda lengua ya que podrán tener conversaciones en inglés con hablantes nativos o con personas que hablen inglés dentro y fuera de la comunidad. Valorarán por ende el aprendizaje de una lengua extranjera como es el inglés para comunicarse y obtener promociones laborales.

\section{¿Por qué décimo y undécimo años de los colegios públicos?}

Los estudiantes de décimo y undécimo años han recibido inglés por tres años anteriormente. Las fortalezas que ellos hayan adquirido durante estos años les proveerán una base lingüística para desarrollar los temas que se incluyen en esta metodología.Al mismo tiempo, a la edad de 16 y 17 años, ya los estudiantes están visualizándose en un mundo laboral debido, sobre todo, a la crisis económica de muchos hogares donde necesitan llevar o ayudar al sostenimiento de la familia. Ya han trazado sus planes futuros al terminar su bachillerato y ellos son conscientes que para obtener cualquier empleo deben saber hablar inglés, al menos, comunicarse.

\section{EVALUACIÓN}

La evaluación en educación es una de las partes más importantes para que el docente y el estudiante puedan determinar los resultados de los procesos en que han estado involucrados. No siempre se obtienen resultados cuantitativos pero los cualitativos también son relevantes para que el proceso educativo sea completo.

Cada vez que se les dice a los estudiantes que van a ser evaluados, ellos reaccionan en forma negativa y no quisieran pasar por ese proceso. Los profesores debemos concientizar a los estudiantes de los diferentes tipos de evaluaciones que se hacen en clase donde se usan diferentes rúbricas. Brookhart y Nitko (2008, p.201) mencionan la analítica, la holística, la general y la que se hace para una actividad específica. Todas ellas vienen en conjunto a ayudar al docente a usar la que considere más provechosa en el proceso que está llevando a cabo. Las cuatro tienen sus ventajas y desventajas; sin embargo, considero que si en algunas actividades, se les da a los estudiantes la opción de que escojan la que ellos creen que es la mejor, que ellos den los diferentes rubros en que quieren ser evaluados y que incluyan la autoevaluación, 
los resultados serían muy productivos (Driscol, 2011, p.4). El docente podría darles una rúbrica que incluya los siguientes parámetros y ellos pueden determinar los indicadores que cada uno debe tener:

1. Estructuras gramaticales

2. Vocabulario

3. Pronunciación

4. Fluidez

5. Contenido

Es así como tanto el alumnado como el docente coexiste en el ámbito evaluativo que por lo general causa mucha ansiedad. Tomar en cuenta a los estudiantes en la evaluación propicia un ambiente mucho más relajado y los resultados pueden ser aún más positivos.

\section{CONCLUSIONES}

Lo propuesto en este artículo no es la solución definitiva para corregir las deficiencias en la destreza oral de la lengua inglesa de los estudiantes graduados de colegios públicos. Los contenidos de los programas de Inglés del Ministerio de Educación, deben servir como base para desarrollar las situaciones aquí sugeridas.

Actualmente, los profesores de décimo y undécimo años dedican su tiempo en clase a proveer a los estudiantes con las estrategias de lectura necesarias para que desarrollen un examen de lectura y puedan obtener su bachillerato. Esta propuesta pretende reforzar dicho objetivo primordial para los profesores y alumnos mediante el desarrollo de los temas en forma oral. Se podría decir que los estudiantes estarían desarrollando al mismo tiempo, la habilidad oral y de comprensión escrita de la lengua inglesa.

Podría verse como una desventaja de esta propuesta, que la destreza oral está limitada a solo los temas que el Ministerio de Educación ofrece en sus programas. Sin embargo, el docente puede ir más allá de esos temas dependiendo de las necesidades de los estudiantes. Si es así, no es un gran problema porque estaríamos logrando más con esto que con darles lecturas con el único objetivo de desarrollar la destreza de la comprensión de lectura. 
La obtención del título de bachiller es una necesidad, especialmente, en una población estudiantil de colegios públicos que necesita salir al campo laboral para llevar el sustento a la familia, o bien, para llenar necesidades básicas personales, como es la de seguir estudiando.

Este método le provee a los graduandos las herramientas necesarias para que puedan enfrentarse a los retos de trabajos como centros de servicio, bien llamados "call-centers", donde se solicitan jóvenes activos y con conocimientos en inglés para contestar llamadas y atender público en hoteles y otras atracciones turísticas de nuestro país.

A través de estas situaciones orales, los estudiantes adquieren una herramienta más cuya finalidad es desarrollar la lengua inglesa. Además de ser un gran paso para que tengan éxito en las entrevistas de centros de servicio, hoteles $u$ otros y logren integrarse al mercado laboral contribuyendo así al crecimiento profesional personal y del país.

\section{Referencias bibliográficas}

Barraja-Rohan, A.M. (2011). Using Conversation Analysis in the Second Language Classroom to Teach Interactional Competence U.S.A : SAGE Publications.

Brookhart .S. y Nitko, A. (2008). Assessment and Grading in Classrooms. NJ: Pearson Education.

Driscol, A. (2011). Constructing Useful and High Quality Rubrics Recuperado de ww.elac.edu/departments/slo/doc/Constructing_Rubrics.pdf

Finocchiaro, M. y Brumfit, C. (1983). The Functional-Notional Approach From Theory to Practice. Oxford: Oxford University Press.

Johnston, W. B. (1991): Global work force 2000: The new world labor market. Harvard Business Review, 69 (2) ,115-27 Recuperado de la Base de Datos ERIC (EJ425238).

Nation, I. S. P. (2001) Learning Vocabulary in Another Language. Cambridge University Press: Cambridge.

Mc Thighe, J. y O'Connor, K. (2009). Seven Practices for Effective Learning.Wadsworth, Canada: Cengage Learning.

MEP. Costa Rica Multilingüe. (2012). Estrategia Siglo XXI: Conocimiento e Innovación para el desarrollo de Costa Rica. Recuperado de http://www.estrategia.cr/es/propiciar-cambio/educacion/ 
Ministerio de Educación Pública La política educativa hacia el siglo XXI. La politica educativa hacia el siglo XXI. (2013). Recuperado de http://www.estadonacion.or.cr/files/biblioteca_virtual/educacion/003/Chavarria_2010_Politica_Educativa_Siglo_XXI.pdf Quintana, H. (1998). Integración Curricular y Globalización. Recuperado de http://ofdp_rd.tripod.com/encuentro/ponencias/hquintana.html

Reyes, A. (2004-2005). Utilidad del campo léxico para la enseñanza de vocabulario en lenguas extranjeras: El campo léxico para describir habilidad mental en inglés. Relingüistica Aplicada, 2, 1-9. Recuperado de http://relinguistica.azc.uam.mx/no002/articulos/ Reling-02_UtilidadCampoLexico.pdf

Schmidth, R.( 1990). The role of consciousness in second language learning. AppliedLinguistics,11(2),129-158. Recuperado de http:// nflrc.hawaii.edu/PDFs/SCHMIDT\%20The\%20role\%20of\%20 consciousness $\% 20 \mathrm{in} \% 20$ second $\% 20$ language $\%$ 20learning.pdf

Zimmerman, C. B. (1997) Do Reading and Interactive Vocabulary Instruction Make a Difference? An Empirical Study? Tesol Quarterly, 31, (1), 121-140. Recuperado de http://www.jstor.org/ discover $/ 10.2307 / 3587978$ ?uid $=2 \&$ uid $=4 \&$ sid $=2110412196$ 


\section{APÉNDICE A}

\section{Contenidos $10^{\circ}$ Año}

1. Achievements of our national athletes

2. Costa Rican Art, music and crafts

3. Costa Rican typical food

4. Holidays and celebrations in Costa Rica and some celebrations in English speaking countries

5. Causes and effects of natural resources misuse

6. Tourist attractions offered by Costa Rican communities

7. Common illnesses and new diseases and epidemics

8. Our Democratic Tradition

9. Careers, jobs and lifestyles

\section{Contenidos $11^{\circ}$ Año}

1. Types of food, eating habits and behaviours at the table

2. Tourists aspects worldwide

3. Job demand in Costa Rica Careers

4. Science and technology

5. Morals and values

6. Gender : Men's and Women's roles

7. Senior citizens, minority groups

8. Mass media and communications 


\section{APENDICE B}

\section{Achievements of our national athletes}

\section{Pre-activity}

Video: http://www.youtube.com/watch?v=1QqrwVkOEmQ

Nery Brenes le da la primera medalla de oro a Costa Rica Lectura: http://en.wikipedia.org/wiki/Nery_Brenes

\section{VOCABULARY}

$\begin{array}{lll}\text { Competition } & & \text { honor of representing } \\ \text { the Olympic } & & \text { race walk } \\ \text { runners: } & \text { Gabriela Traña, Nery Brenes and Allan Segura } & \text { heavy weight } \\ \text { mountain biker: } & \text { Federico Ramirez } & \text { light weight } \\ \text { road cyclist: } & \text { Henry Raabe } & \text { distance } \\ \text { martial artist: } & \text { Kristopher Moitland } & \text { track and Road } \\ \text { swimmers: } & \text { Quesada and Mario Montoya } & \text { hurdle } \\ \text { triathlete: } & \text { Leonardo Chacón } & \\ \text { Cycling } & \text { Paolo César Montoya } & \\ \text { Free-style } & & \text { jumping } \\ \text { Compete } & & \text { throwing } \\ \text { Committee } & & \text { medals } \\ \text { sprint } & & \\ \text { running and relay baton } & \end{array}$




\section{USEFUL LANGUAGE}

$\mathrm{He} / \mathrm{she}$ won gold/silver/ bronze gold (s) in

$\mathrm{He} /$ she was born in . He/she is years old. He/she/ has children.

$\mathrm{He} /$ she has lived in for

$\mathrm{He} /$ she has been training for

She/he must be on a -diet that includes

The company that sponsors him/her is I don't know

I know that

I heard /read the news about

The government should encourage/help/ give

Let's go to the airport. Bring flags, posters, music.

What kind of $?$

How can/will $?$

swimming/hiking, mountain biking, playing soccer

\section{SITUATION 1 Achievements of our national athletes}

Talk to your partner about the gold medals that José Nery Brenes got lately. Discuss his discipline and endurance towards athletics.

\section{SITUATION 2 Achievements of our national athletes}

Plan a warm welcome at the airport for those soccer players or athletes that won some medals abroad. Talk about music, posters, flags, journalists and so on. 


\section{Costa Rican Art, music and crafts}

\section{Pre-activity}

Video: http://www.youtube.com/watch?v=RtG8z49zZH8 Costa Rican Music Performance in Tortuga Island

Lectura: http://costarica-information.com/about-costa-rica/art-history/ costa-rica-art/music

\section{Costa Rican Art, music and crafts}

\begin{tabular}{|c|c|c|}
\hline \multicolumn{3}{|l|}{ Vocabulary } \\
\hline Costa Rica’s art history & the national fine arts & frame \\
\hline Escuela Nacional de Bellas Artes & architect Works & gesso \\
\hline painter & private projects $\mathrm{f}$ & orefathers \\
\hline engraver & artists & mentors \\
\hline muralist & passionate & vast production \\
\hline granite & wood & carvings \\
\hline illustrator & sculptor & water/oil paintings \\
\hline exhibitions & display/show & stone \\
\hline background & prize & museums \\
\hline art work & workshop & canvass \\
\hline a sanctuary & indigenous & preserve /portrait \\
\hline singer & master & piece \\
\hline \multicolumn{3}{|c|}{ Costa Rican forefathers and mentors: } \\
\hline \multicolumn{3}{|l|}{ Francisco "Paco" Amighetti } \\
\hline \multicolumn{3}{|l|}{ Crisanto Badilla } \\
\hline \multicolumn{3}{|l|}{ Fadrique González } \\
\hline \multicolumn{3}{|l|}{ Teodorico Quirós } \\
\hline \multicolumn{3}{|l|}{ Hugo Sánchez } \\
\hline \multicolumn{3}{|l|}{ Juan Manuel Sánchez } \\
\hline Juan Rafael Chacón & & \\
\hline
\end{tabular}




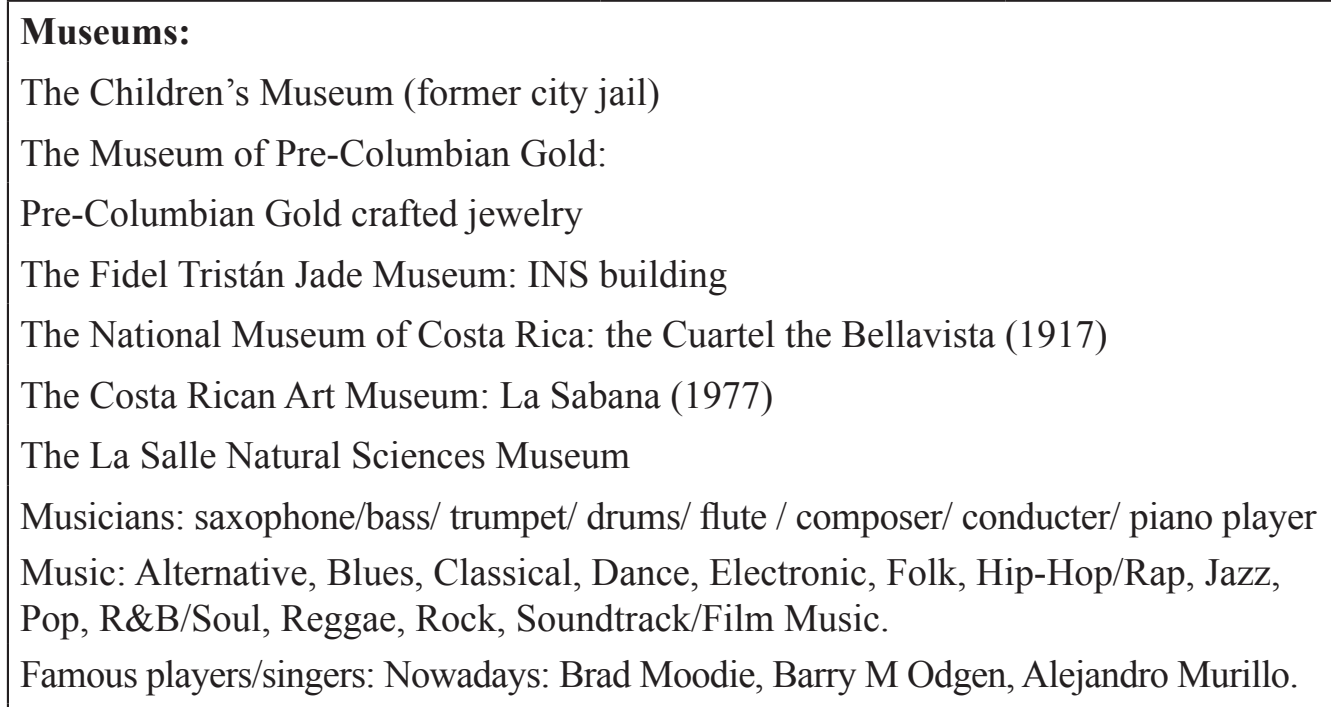

The Melico Salazar Theatre is located downtown. It maintains a full schedule of concerts, recitals, and plays. Construction began 8 February 1927, and was completed 7 October 1928.

Opened: 7 February 1928

The National Theater in Costa Rica was created in 1897. Constructed in the late 19th century, when San Jose's population was only around 19,000 people, the theatre presented many private performances. As well as having performances several times a week, the theatre is a tourist attraction. Performances by the National Symphonic Orchestra (NSO) take place as part of orchestra's regular season, and include both Costa Rican and foreign composers. 


\section{USEFUL LANGUAGE}

$\mathrm{He} / \mathrm{she}$ was born in _ in

She/he is/was recognized as

She/he won an award of

It's located in

You can buy the ticket in . Or just call to

It was founded in

She/he is famous for

In my opinion,

I agree with

When was founded/created?

I disagree with

Have you been to a concert in the National Theater/Melico Salazar Theater?

The most exciting experience is

\section{SITUATION 1 Costa Rican art, music and crafts}

Talk to your partner about the importance of art as a reflection of our culture. Discuss how some painters depict traditions, colors and designs of our culture.

\section{SITUATION 2 Costa Rican art, music and crafts}

You are at the National Museum. Discuss the work of a sculptor, or painter. (the professor will give you a picture or photo of an art work) Ask your partner about important features of his/her work. Talk about color and size of the artwork. 


\section{APENDICE C}

\section{Pre-activity Science and Technology}

\section{Lecturas: What's Intel}

http://download.intel.com/education/worldahead/Costa_Rica_Intel_ Teach_Program.pdf

English teacher's helper: http://www.globalpost.com/dispatch/ education/100421/costa-rica-english-classroom

\section{Science and Technology}

\section{VOCABULARY}

\begin{tabular}{|c|c|}
\hline Laptop & $\begin{array}{l}\text { Earth's atmosphere (such as the cosmic } \\
\text { background radiation). }\end{array}$ \\
\hline telephone & evolution, physics \\
\hline E-mail support & chemistry \\
\hline customers & meteorology \\
\hline professionalism & motion \\
\hline rapid response-time & formation \\
\hline server location & research \\
\hline global plate tectonics & browse \\
\hline mantle rocks & download \\
\hline knowledge & delete \\
\hline scientific method, & character \\
\hline laws and theories & software \\
\hline Astronomy & hardware \\
\hline natural science & design programs \\
\hline $\begin{array}{l}\text { celestial objects (such as stars, planets, } \\
\text { comets, nebulae, star clusters and } \\
\text { galaxies) phenomena }\end{array}$ & use images \\
\hline
\end{tabular}




\begin{tabular}{|l|l|}
\hline implementation & $\begin{array}{l}\text { Hard drive } \\
\text { requirements } \\
\text { gigabytes / megabytes } \\
\text { artifacts } \\
\text { keys/locks/fasteners/keyboard/ wire/ } \\
\text { speakers / printer/ camera/ } \\
\text { circuit board } \\
\text { processor } \\
\text { memory/RAM } \\
\text { motherboard } \\
\text { graphics card } \\
\text { network card } \\
\text { modem }\end{array}$
\end{tabular}$\quad$\begin{tabular}{l} 
pixels \\
resolution \\
plasma screen/display \\
digital machine/camera \\
mobile devices \\
attachment \\
reply \\
forward \\
cell phones /IPod/ \\
data traveler (Kingston) \\
flash memory \\
\hline
\end{tabular}

\section{SITUATION 1 Science and Technology}

Your parents will buy a computer for you. Talk about the best purchase, what you want, the brand, the capacity and other hardware you would like to have.

\section{SITUATION 2 Science and Technology}

Discuss advantages and disadvantages of having a laptop/ a cell phone, or an iPod. Talk about software, hardware, prices, ease of comfort, range of products \& products usage 


\section{Morals and values}

\section{Pre-activity}

Lectura: http://changingminds.org/explanations/values/values_morals_ ethics.htm

\section{VOCABULARY}

\begin{tabular}{|l|}
\hline insult \\
offense \\
violence \\
threaten \\
thieve/burglar \\
punishment \\
sentence \\
moral responsibility \\
loyalty \\
virtue \\
ethics \\
philosophy \\
tendency \\
respect \\
requirement \\
qualities \\
qualifications \\
endurance \\
love for the country \\
obey the moral demands \\
learn to respect other's way of thinking \\
human well-being
\end{tabular}

behavior

benevolence

objectivity

subjectivity

perfection

empathy

autonomy

rationality

control of one's feelings

understanding of what it's right/wrong

creativity

appreciation

controversial

self-doubt

skepticism

honesty

sincerity

faithful

irrational

persuasive power

helpful

what is really valuable in our lives 


\section{USEFUL LANGUAGE}

Do you consider $?$

I consider myself

I disagree/ agree with

I believe in

What's your attitude toward (s) ?

I don't think is true/ false.

I'm/ not familiar with this term, but I think

My thinking here has been immediately influenced by

What values are taken into account by employers when you want to get a job?

What do you think is the most important value in a friend relationship?

\section{SITUATION 1 Morals and values}

Talk about some of the most important values a person should have to be a good worker in a company. Discuss the different problems a company may have if its employees. Mention a specific case you know.

\section{SITUATION 2 Morals and values}

Discuss the importance of learning morals and values when you are a child. Tell your partner what values are the ones that have helped you be a good student, friend, son/daughter/ neighbour. Ask him/her about his/hers. 